\title{
Engineering cancer vaccines using stimuli-responsive biomaterials
}

\author{
Yu Zhao ${ }^{1}$, Yugang Guo ${ }^{1}$, and Li Tang ${ }^{1,2}(\varangle)$ \\ ${ }^{1}$ Institute of Bioengineering, École polytechnique fédérale de Lausanne (EPFL), Lausanne, CH-1015, Switzerland \\ ${ }^{2}$ Institute of Materials Science \& Engineering, École polytechnique fédérale de Lausanne (EPFL), Lausanne, CH-1015, Switzerland
}

\author{
Received: 26 May 2018 \\ Revised: 27 July 2018 \\ Accepted: 28 July 2018 \\ (c) Tsinghua University Press \\ and Springer-Verlag $\mathrm{GmbH}$ \\ Germany, part of Springer \\ Nature 2018

\section{KEYWORDS} \\ cancer vaccine, \\ stimuli-responsive, \\ biomaterial, \\ nanoparticle, \\ immune response, \\ lymph node targeting, \\ cross-presentation
}

\begin{abstract}
Cancer vaccines aimed at expanding the pool or increasing the activity of tumor-specific $\mathrm{T}$ cells against malignancies is an important immunotherapy modality that has been extensively pursued in the past decades. However, the clinical efficacy of cancer vaccines remains modest in comparison to other immunotherapies, such as checkpoint blockade and adoptive $\mathrm{T}$ cell therapy. This unsatisfactory performance is likely due to the suboptimal selection of tumor antigens for vaccine and inefficient delivery platform. Recently, vaccines designed to target cancer neoantigens have shown marked promise in both preclinical and early clinical studies. However, enormous challenges need to be overcome to develop a highly efficient and safe delivery strategy for targeting cancer vaccines to professional antigen-presenting cells and eliciting optimized immune response against cancers. To meet these challenges, biomaterials, particularly biomaterials that are designed to respond to certain environmental stimuli, termed as stimuli-responsive biomaterials, are being actively developed to precisely manipulate the trafficking and release of cancer vaccines in vivo for enhanced therapeutic efficacy and safety. In this mini review, we provide a brief overview of the recent advances in applying stimuli-responsive biomaterials in enhancing non-cellular cancer vaccines while focusing on the chemistry and material design with varied responsiveness. We also discuss the present challenges and opportunities in the field and provide a perspective for future directions.
\end{abstract}

\section{Introduction}

Cancer vaccine as an active immunotherapy that is designed to elicit robust immune response against cancer cells has been long pursued in the last few decades [1]. Despite successful examples of vaccines against many infectious diseases, highly effective and safe cancer vaccines have yet to be developed. To date, only a couple of prophylactic cancer vaccines against virus-related cancers, e.g., cervical cancer associated

Address correspondence to li.tang@epfl.ch 
with human papillomavirus, are available in the market. And only one therapeutic cancer vaccine, i.e., Provenge ${ }^{\circledR}$, a dendritic cell (DC)-based vaccine, has been approved by U.S. Food and Drug Administration (FDA) for the treatment of prostate cancer [2]. Thus far, only modest clinical efficacy has been achieved with various therapeutic cancer vaccines. The unsatisfactory performance of current cancer vaccines can be attributed to two major obstacles: (1) the difficulty in selecting a tumor-specific and immunogenic antigen as the ideal target; (2) the ineffective and uncontrolled delivery of vaccine components at tissue and cell levels. Recent advances in neoantigen-based cancer vaccines will likely provide a solution for antigen selection to design more potent cancer vaccines [3]. However, it remains challenging to develop an efficient and safe delivery strategy to target cancer vaccines against neoantigens to professional antigen-presenting cells (APCs) and elicit optimized anticancer immune response [4].

In the past decades, extensive investigation on biomaterials has achieved tremendous success in constructing controlled drug delivery systems [5]. As a highly interdisciplinary contribution across chemistry, physics, biology, and medicine, biomaterials cover a vast collection, comprising extremely diverse chemical structures (e.g., synthetic polymers, biomacromolecules, and inorganic crystals), various morphologies (e.g., soluble conjugates, nano-/micro-particles, and bulk materials), and numerous biological functions (e.g., biocompatibility, biodegradability, cargo protection, selective targeting, and controlled release) [6-8]. Benefiting from the drastically flexible design, a huge library of biomaterials as drug carriers has been established to meet the demands in complicated and diverse physiological conditions. Owing to the immense success in drug delivery, immunologists and material scientists also attempted utilizing biomaterials to overcome the hurdles in vaccine delivery. To date, novel cancer vaccines based on advanced biomaterials, particularly nanoparticles (NPs), have already exhibited high promise in eliciting immune response against cancers in preclinical and clinical studies. Such efforts have been comprehensively reviewed by a plenty of refined articles [9-11].

Among others, "smart" biomaterials with tailordesigned stimuli-responsiveness have been considered as one of the most promising carrier for advanced drug delivery design [12]. By advantageous usage of diverse internal stimuli in the physiological microenvironment, e.g., $\mathrm{pH}$, redox potential, temperature, enzyme, mechanical force, and some easily-controlled external triggers, e.g., light and ultrasound, stimuliresponsive biomaterials have been developed to achieve highly controlled delivery and release of various drug cargos, including vaccine components [13]. This particular category of biomaterials provides the unique opportunity for precise spatiotemporal control of the delivery process, which is of great significance in improving the efficacy and minimizing the "off-target" toxicity of drugs. Investigation on stimuli-responsive drug delivery systems has also been extensively reviewed by numerous outstanding review articles [14, 15]. However, to the best of our knowledge, a comprehensive review on applying stimuli-responsive biomaterials for cancer vaccine delivery is still lacking. In this mini review, we focus on the responsive delivery of non-cellular subunit cancer vaccines. We introduce several representative design strategies of stimuli-responsive biomaterials in response to different triggers for enhancing the efficacy of subunit cancer vaccines by highlighting the recent advances in the field, discuss the pros and cons of each approach, and present our prospective on the future challenges and opportunities.

\section{Designing an effective cancer vaccine}

Many excellent reviews have well-presented the design criteria of a successful cancer vaccine $[16,17]$. Here, we briefly summarize the general considerations in designing a non-cellular cancer vaccine and discuss the challenges in vaccine delivery. A potent immune response elicited by vaccines relies on the activation of adaptive immune system, comprising $\mathrm{B}$ and $\mathrm{T}$ lymphocytes. B cells are key factors in defending ourselves against infectious invasions through the humoral immune response, i.e., secreting antibodies to neutralize exogenous pathogens. $\mathrm{CD}^{+} \mathrm{T}$ cells facilitate the activation of other immune cells by producing cell-signaling cytokines, whereas $\mathrm{CD}^{+}$ cytotoxic T lymphocytes (CTLs) play a significant role in inducing apoptosis of malignant or infected 
cells, a process known as cellular immune response [18]. It has been generally accepted that it is critical to elicit potent $\mathrm{CD} 8^{+} \mathrm{T}$ cell response by an anticancer vaccine for direct cancer cell killing $[17,19]$. Upon vaccination, APCs acquire and internalize the antigens through endocytosis, followed by two major pathways for antigen processing and presentation [20]: (1) Antigens trapped in endosomes are degraded by protease and directly loaded on major histocompatibility complex (MHC) Class II molecules for presentation to $\mathrm{CD}^{+}$helper $\mathrm{T}$ cells, and, generally, this pathway through MHC Class II molecules is a default for subunit vaccines; (2) antigens that have escaped from endosomes/lysosomes and entered the cytosol are processed by proteasomes to generate antigen-specific epitopes that bind to MHC Class I receptors on the endoplasmic reticulum (ER) and are subsequently expressed on the cell surface for antigen presentation to $\mathrm{CD}^{+} \mathrm{T}$ cells. This process is known as crosspresentation and is critical for eliciting $\mathrm{CD}^{+} \mathrm{T}$ cell immune response. Therefore, efficient cross-presentation is the key to a potent cancer vaccine for robust $\mathrm{T}$ cell immunity (Scheme 1).

Cancer vaccines typically contain two rudimentary components, (1) subunit antigen, such as proteins, peptides, or DNA/mRNA encoding the epitopes,

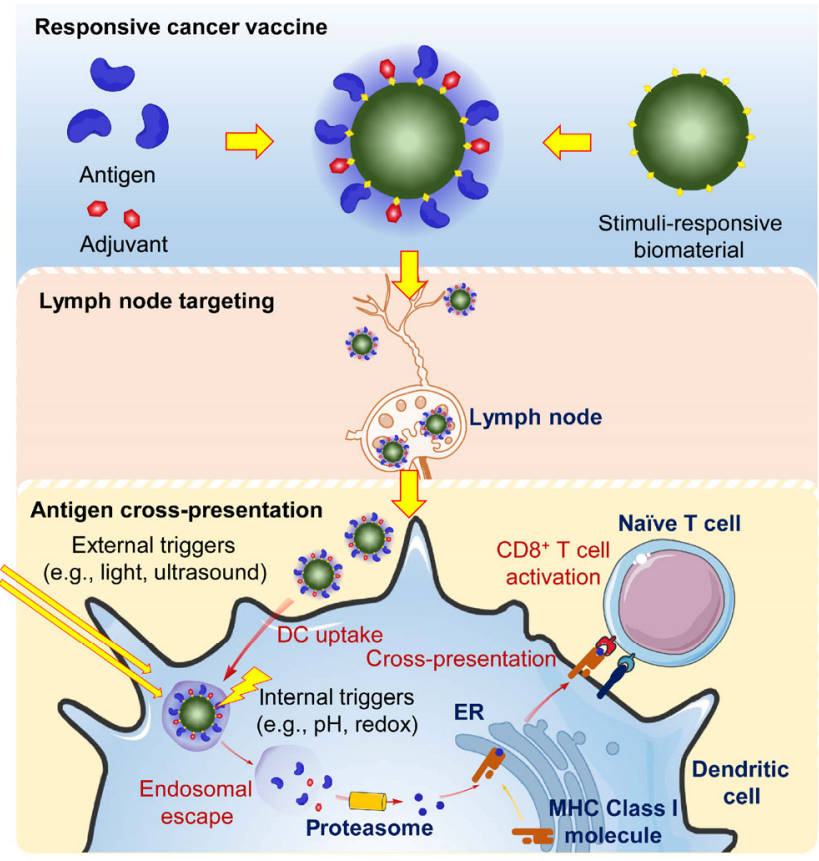

Scheme 1 Designing principles for an effective cancer vaccine. which is derived from tumor or tumor-related tissues; (2) adjuvant, a molecule or material that promotes immune response against the co-administered antigen. A subunit antigen is typically poorly immunogenic and must be combined with adjuvants that activate APCs in a cancer vaccine. Modern molecular adjuvants include agonists for pattern-recognition receptors, e.g., toll-like receptors (TLRs) [21, 22]. Thus, delivery of both the antigen and adjuvant to the lymphoid organs and APCs is necessary for an effective cancer vaccine.

Together, to make a cancer vaccine effective, one must address two major challenges in delivery simultaneous: (1) targeting vaccine components to the immune system and APCs; (2) promoting antigen cross-presentation. The former is a tissue-level delivery, whereas the latter is an intracellular delivery. Cancer vaccines administered primarily through parenteral injections should be targeted to secondary lymphoid organs, such as lymph nodes (LNs) or mucosaassociated lymphoid tissues (MALTs), for the most efficient antigen presentation as these lymphoid organs contain a much higher concentration of DCs than peripheral tissues and are the locations where naïve T cells are activated by APCs. However, soluble antigens and molecular adjuvants typically tend to disseminate into blood and are cleared rapidly because of the small size, which results in extremely low vaccine efficiency and high risk of systemic toxicities. Additional challenges include poor vaccine capture by APCs and nearly exclusive loading onto MHC Class II molecules of subunit vaccines leading to insufficient $\mathrm{CD}^{+} \mathrm{T}$ cell response, which is critical for cancer cell eradication. An effective and versatile delivery platform is highly desired for cancer vaccines to overcome these challenges for eliciting potent anticancer immune response.

\section{Controlling the delivery of cancer vaccine with stimuli-responsive biomaterials}

Stimuli-responsive biomaterials have exhibited great promise in addressing delivery challenges of cancer vaccines at both tissue and cell levels. Tailor-designed biomaterials are optimized through flexible chemistry design for high sensitivity to one or more specific triggers, ensuring selective antigen processing by the 
immune system and controlled immune response. Here, we highlight some recent advances in applying responsive biomaterials to cancer vaccines for enhanced efficiency and safety and discuss their pros and cons in this section (Table 1). Depending on the functions, we categorize these responsive biomaterials into those used for enhancing LN and APC targeting and those used for enhancing cross- presentation.

\subsection{Responsive biomaterials for enhancing LN and APC targeting}

Most responsive biomaterials so far developed for cancer vaccine delivery have been designed to target the components of vaccines to LNs as an ideal lymphoid organ to elicit anticancer $\mathrm{CD} 8^{+} \mathrm{T}$ cell response. It has been shown that NPs with a diameter in the range of $9-100 \mathrm{~nm}$ preferentially traffic to LNs spontaneously [23, 24], whereas NPs larger than $100 \mathrm{~nm}$ rely on the capture and subsequent transportation by resident APCs. Therefore, the size of particulate biomaterials has to be optimized to facilitate the most effective delivery of vaccine components to LNs [22]. To further increase internalization by professional APCs, particularly DCs, the surface of NP-based delivery systems are decorated with DC-targeting moieties, such as anti-CD11c antibody and mannose [25, 26]. Together, nanomaterials with optimized size and surface modification could be utilized to enhance targeted delivery of both antigen and adjuvant to LNs and APCs $[9,27]$. Certain responsiveness of a biomaterial, in addition to the properties of size or surface modification, is also explored for enhanced targeting to LNs and internalization by APCs. In an elegant example, Lynn et al. developed an in situ self-

Table 1 Advances in engineering cancer vaccines with stimuli-responsive biomaterials

\begin{tabular}{|c|c|c|c|}
\hline Stimuli & Responsive chemical structure & Delivery system & Representative Ref. \\
\hline \multicolumn{4}{|c|}{ Responsive biomaterials for enhanced $\mathrm{LN}^{\mathrm{a}}$ targeting } \\
\hline Temperature & NIPAM $^{b}$ & Vaccine-polymer conjugate & {$[28]$} \\
\hline \multicolumn{4}{|c|}{ Responsive biomaterials for enhanced cross-presentation } \\
\hline \multirow{9}{*}{$\begin{array}{l}\text { Acidic environment } \\
\qquad(\mathrm{pH})\end{array}$} & Acetal bond & Crosslinked polymeric $\mathrm{NP}^{\mathrm{c}}$ & {$[29]$} \\
\hline & Coordination bond & $\mathrm{MOF}^{\mathrm{d}}$ & {$[30]$} \\
\hline & Carboxyl group & Antigen-polymer conjugate & [31] \\
\hline & & Micelle & {$[32]$} \\
\hline & & Liposome & {$[33]$} \\
\hline & $\mathrm{NH}_{4} \mathrm{HCO}_{3}$ & PLGA $^{\mathrm{e}} \mathrm{NP}$ & {$[34]$} \\
\hline & Tertiary amine & Polymeric NP & {$[35]$} \\
\hline & Fusogenic peptide & Tumor cell-derived exosome & [36] \\
\hline & & Self-assembly micelle & [46] \\
\hline \multirow{4}{*}{$\begin{array}{l}\text { Reductive environment } \\
\text { (redox) }\end{array}$} & Disulfide bond & Polymeric NP & [37] \\
\hline & & Vaccine conjugate & {$[38,39]$} \\
\hline & & MOF & [40] \\
\hline & & Antigen/adjuvant NP & [41] \\
\hline \multirow{4}{*}{$\begin{array}{c}\text { Light } \\
\left(\text { Vis }^{f} \text { or } \mathrm{NIR}^{\mathrm{g}}\right)\end{array}$} & TPCS $2 \mathrm{a}^{\mathrm{h}}$ & Antigen-polymer conjugate & {$[42]$} \\
\hline & & Polymeric NP & {$[43]$} \\
\hline & PheoA $^{\mathrm{i}}$ & Polymeric NP & {$[44]$} \\
\hline & Gold NP & Inorganic NP & [45] \\
\hline Ultrasound & Bubble lipoplex & Liposome & {$[46,47]$} \\
\hline
\end{tabular}

${ }^{a} \mathrm{LN}$, lymph node; ${ }^{b} \mathrm{NIPAM}, \mathrm{N}$-isopropylacrylamide; ${ }^{\mathrm{N} P}$, nanoparticle; ${ }^{\mathrm{d}} \mathrm{MOF}$, metal-organic framework; ${ }^{\mathrm{e}} \mathrm{PLGA}$, poly(lactic-co-glycolic acid); ${ }^{\mathrm{f}}$ Vis, visible; ${ }^{\mathrm{g}} \mathrm{NIR}$, near infrared; ${ }^{\mathrm{h}} \mathrm{TPCS} 2 \mathrm{a}$, tetraphenyl chlorine disulfonate; ${ }^{\mathrm{i}}$ PheoA, pheophorbide A. 
assembling temperature-responsive vaccine-polymer conjugate [28]. An N-isopropylacrylamide (NIPAM)based thermoresponsive copolymer p[(NIPAM)-co(Ma-Ahx-TT)] conjugated with TLR-7/8 agonists was modified with a coil peptide, which facilitated the formation of a heterodimer with a coil-peptide-antigen fusion protein (Fig. 1(a)). The thermoresponsive heterodimer remained soluble at room temperature and self-assembled into immunogenic particles at physiologic temperature upon injection (Fig. 1(b)). This tailor-designed delivery system combined the advantages of well-defined chemical structure and high stability, which are typical only for soluble antigens and adjuvants, and enhanced LN targeting and APC

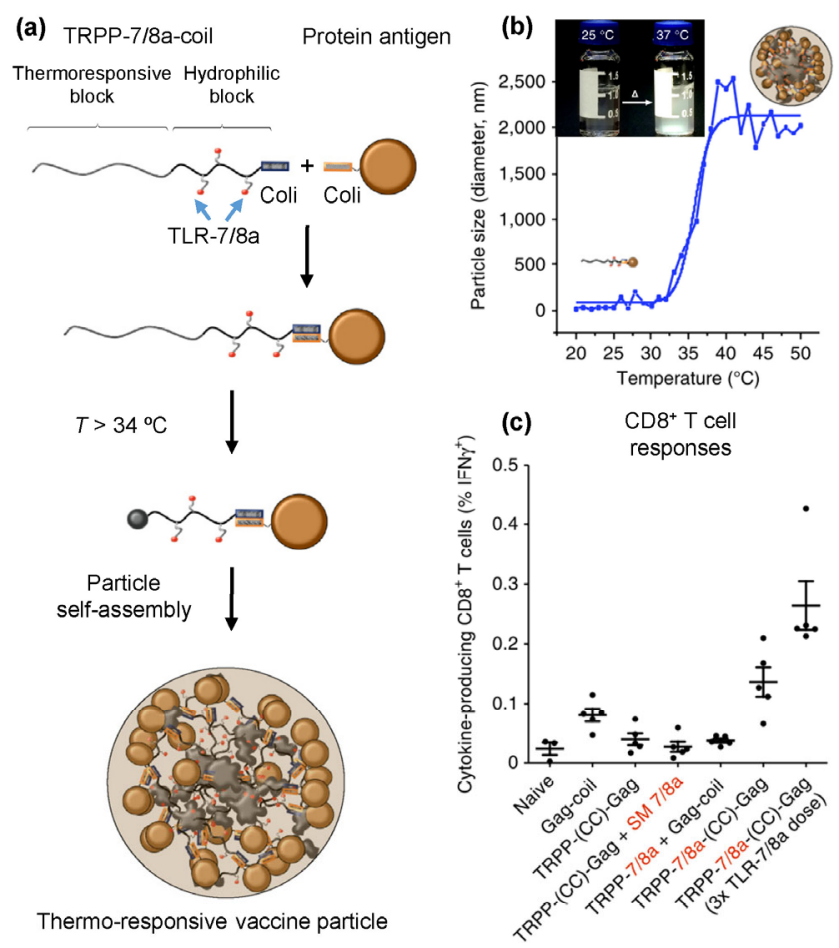

Figure 1 Thermoresponsive polymer-TLR-7/8 agonist (TRPP7/8a) conjugate linked with protein antigen self-assembled into vaccine particles for enhanced lymph node retention and APC internalization. (a) TRPP-7/8a modified with a coil peptide, which forms heterodimers with a recombinant HIV Gag-coil fusion protein, to form TRPP-7/8a-(CC)-Gag, self-assembled into the vaccine particle in vivo at the physiological temperature. (b) Temperature-dependent particle formation measured by dynamic light scatting. (c) Antigen-specific IFN- $\gamma$-producing $\mathrm{CD}^{+} \mathrm{T}$ cells in the mixed splenocyte cultures from immunized BALB/c mice. Splenocytes were stimulated in vitro with an HIV Gag peptide pool. Reproduced with permission from Ref. [28], (C) Nature Publishing Group 2015. internalization capability, superiorities typical only for particulate vaccines. Vaccinated BALB/c mice with this thermoresponsive vaccine exhibited markedly enhanced activation and proliferation of antigen-specific $\mathrm{CD} 4^{+}$ and $\mathrm{CD}^{+} \mathrm{T}$ cells and more potent antibody response compared to that by the non-responsive counterpart (Fig. 1(c)). Despite the wide applications of thermoresponsive biomaterials in other drug delivery systems, such as anticancer drugs, similar attempts on cancer vaccine delivery with thermoresponsive biomaterials are relatively rare so far. This study provides a promising example in utilizing heat-triggered selfassembly to enhance LN targeting.

Several strategies have been developed to date to target cancer vaccines to LNs, such as size control, albumin hijacking, and direct intranodal injection [48-50]. However, there are still rather limited efforts thus far for designing responsive biomaterials to facilitate LN targeting of cancer vaccines. Given the highly controlled and flexible properties of responsive biomaterials, similar delivery systems can be foreseen to target cancer vaccines to LNs.

\subsection{Responsive biomaterials for enhancing cross- presentation}

\subsection{1 pH-responsive vaccine delivery system}

Endocytic compartments of DCs exhibit mildly acidic $\mathrm{pH}$ compared to that of the extracellular environment and other intracellular compartments. Upon antigen internalization, which is typically through the endocytosis pathway, the vacuolar proton pump is subsequently activated to induce acidification in lysosome, which then fuses with endosomes and facilitates the proteolysis of internalized antigens [51]. The $\mathrm{pH}$ level within endosomes/lysosomes can be as low as 4.5 in late endosomes [52], providing an ideal internal trigger to control antigen release in a $\mathrm{pH}-$ responsive vaccine delivery system. In order to achieve a $\mathrm{pH}$-responsive system for intracellular delivery, strategies dependent on diverse mechanisms, including acid-catalyzed degradation, proton-mediate phase transition, and "proton sponge" effect, have been developed thus far. In fact, $\mathrm{pH}$-responsive biomaterials have been explored most extensively among others for cancer vaccine delivery. 


\subsubsection{1 pH-responsive biomaterials containing acid-labile structures}

Encapsulating vaccine components within acid-labile polymeric NPs is one of the most straightforward strategies for designing $\mathrm{pH}$-responsive cancer vaccines. This type of vaccine carriers relies mostly on acid-sensitive chemical linkers, which remain inert extracellularly at neutral or slightly basic $\mathrm{pH}$ but rapidly disintegrate in acidic cellular compartments. Substantial and instant increase of the local concentration of antigen molecules disrupts the balance of osmotic pressure across the endosomal membrane and facilitates the escape of antigens from the ruptured endosomes. Fréchet group and others pioneered in designing and synthesizing such polymeric NPs for vaccine delivery $[53,54]$. They established a $\mathrm{pH}-$ responsive vaccine delivery platform by embedding acid-labile ketal-derived moieties in the linkers that crosslinked polymer chains to form a microgel. This method is versatile and applicable in various crosslinked biomaterials prepared with different synthetic polymers, such as polyacrylamide derivatives and biodegradable polyurethanes [29, 55]. Utilizing the $\mathrm{pH}$-responsive microgel as a delivery vehicle, the prepared vaccine exhibited highly increased antigen release from the endosome, leading to substantially enhanced cross-priming of $\mathrm{CD}^{+} \mathrm{T}$ cells and their effector functions in the vaccinated mice compared with that by a non-/less-responsive vaccine [56]. These particulate vaccines can be further conjugated with DC-targeting antibodies on the surface, e.g., antiDEC-205 antibody, for enhanced targeting efficiency to DCs $[29,57]$.

Early attempts focused on the delivery of antigenic proteins to DCs with less effort in the targeted delivery of adjuvants. Recently, De Geest group realized the enhanced intercellular release of adjuvant molecules in DCs with an acid-labile delivery system [58]. In their study, an imidazoquinoline derivative, a smallmolecule TLR7/8 agonist, was covalently ligated onto the polymer chain that self-assembled into a polymeric nanogel crosslinked with a ketal-containing linker. The nanogel vaccine exhibited accelerated degradation under endosomal $\mathrm{pH}$ leading to substantially enhanced activity and safety for adjuvant delivery.
The same group next combined the thermo- and $\mathrm{pH}$-responsiveness in one nanogel; this nanogel exhibited enhanced LN targeting and retention as well as the endosomal release of the adjuvants for increased activity [59]. Such dual-responsive system affords the advantages in controlling both tissue-level and intracellular delivery of a cancer vaccine for optimized immune response.

It has been shown in previous studies that the co-delivery of both the antigen and adjuvant is essential for a potent cancer vaccine design as signal 1 and signal 2 are both necessary to activate T cells [60]. Responsive biomaterials have been employed for such co-delivery. For example, Duan et al. demonstrated an acid-degradable metal-organic framework (MOF)based NP through coordinating lanthanide ions and guanine monophosphate (GMP) for the co-delivery of both tumor-associated antigens and adjuvants via encapsulation and surface bounding, respectively (Fig. 2) [30]. Loading of ovalbumin (OVA), a model antigen, to this MOF-based delivery system was achieved via a facile one-pot reaction together with NP preparation; the guanine nucleic acid on the surface facilitated the incorporation of a cytosinephosphate-guanine oligodeoxynucleotide (CpG-ODN) adjuvant, a TLR-9 agonist, through Watson-Crick base pairing (Fig. 2(a)). The size of the MOF-NP was optimized (with a diameter of $30 \mathrm{~nm}$ ) for LN targeting and DC internalization. Rapid particle degradation and antigen release occurred at a $\mathrm{pH}$ of $<5.0$, leading to improved vaccination efficiency and efficacy against OVA-expressing B16 melanoma (B16-OVA) mouse model (Figs. 2(b)-2(d)).

Biomaterials based on acid-labile chemical structures are a straightforward yet versatile approach to achieve $\mathrm{pH}$-responsiveness. Given the vast collection of pH-labile chemical structures, such as the reversible amide bond formed between maleic anhydride and primary amine [61], there are still various ways for enriching the library of responsive biomaterials and, thus, further improving the efficiency of crosspresentation. In addition to NPs that have been studied most extensively, other morphologies of biomaterials, e.g., hydrogel and microneedles, can also be imparted acid-degradability and utilized in designing $\mathrm{pH}$ responsive cancer vaccines $[62,63]$. 

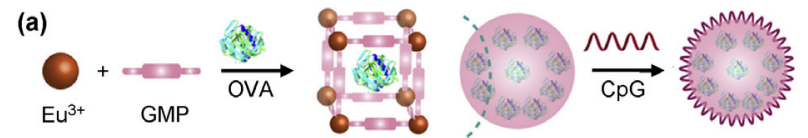

(b)
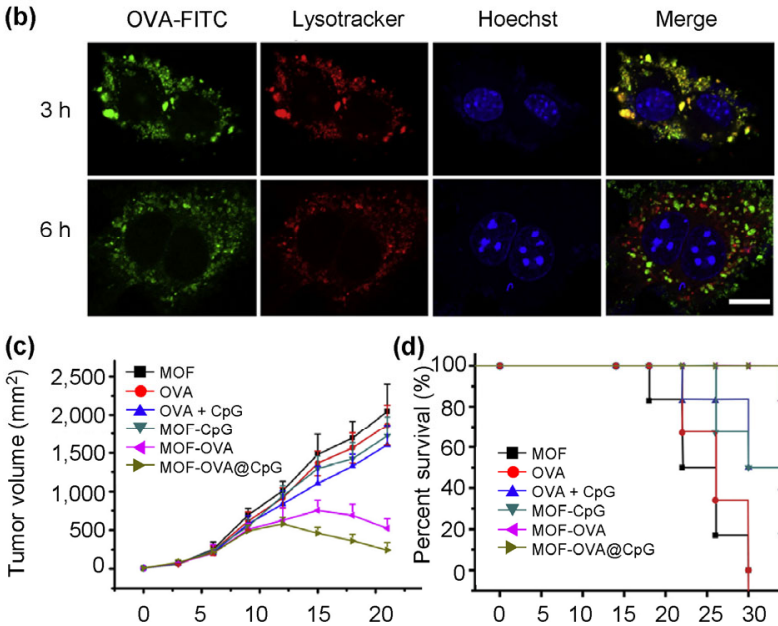

(d)

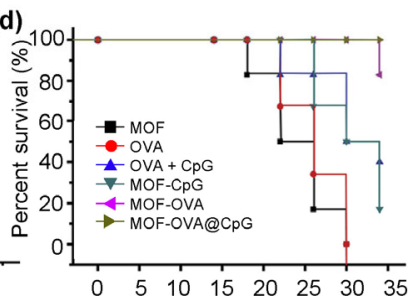

Figure 2 pH-sensitive cancer vaccine delivery system based on MOF. (a) Schematic illustration of the preparation of the model antigen, OVA-loaded MOF vaccines through the "one-step" process. (b) Endosomal escape of OVA-loaded MOF nanoparticulate vaccines labeled with fluorescein isothiocyanate (FITC) in RAW264.7 cells analyzed by confocal laser scanning microscopy. Scale bar = $10 \mu \mathrm{m}$. (c) Inhibition of B16-OVA tumor growth in mice vaccinated with OVA- and CpG-loaded MOF nanovaccine (MOF-OVA@CpG). (d) Survival curves of mice vaccinated with different formulations. Reproduced with permission from Ref. [30], (c) Elseiver 2017.

\subsubsection{2 pH-responsive biomaterials based on acid-triggered phase transition}

Non-degradable pH-responsive biomaterials, such as polycarboxylic acids and charged peptides, are also extensively exploited for vaccine delivery. These pH-responsive biomaterials exhibit significant phase transition in acidic environment due to the increase in hydrophobicity upon protonation. The hydrophobic interaction between the protonated polymer and hydrophobic domain of phospholipids disrupts the lipid membrane of the endosome and, thus, enhances endosomal escape of the vaccine components [64]. For example, Stayton and colleagues investigated a comprehensive collection of carboxyl-containing polymers for vaccine delivery. A model antigenpoly(propylacrylic acid) (PPAA) conjugate prepared by this group exhibited 8-fold increase in antigenspecific $\mathrm{CD}^{+} \mathrm{T}$ cell proliferation and markedly improved tumor-free survival of mice bearing EG.7OVA tumors compared to that by the non-responsive counterpart [31, 65]. Moreover, they developed several similar delivery systems, including self-assembly micelles composed of tailor-designed amphiphilic block copolymers $[32,66]$. A high number of biomaterials with the property of acid-triggered phase transition have been developed for $\mathrm{pH}$-responsive vaccine delivery owing to the facile chemical synthesis of this kind of material. However, it is worth noting that phase transition of vaccines carriers may only facilitate endosomal escape of cancer vaccines, but not necessarily the release of encapsulated antigens and adjuvants from the carriers. Therefore, responsive degradability, such as a cleavable disulfide bond, is often imparted to this kind of biomaterial to accelerate the release of the vaccine components from the carriers once they have reached the cytosol of APCs [32].

In addition to polycarboxylic acids and their derivatives, fusogenic biomaterials exhibiting $\mathrm{pH}$ mediated phase transition have also been employed to facilitate endosomal disruption and intracellular delivery of cancer vaccines $[67,68]$. Once internalized into the acidic endosomes, such materials alter their tertiary structure from a random coil to $\alpha$-helix, leading to pore formation in the endosome membrane through $\alpha$-helix-lipid association. For example, Yuba et al. developed a series of liposome-based $\mathrm{pH}$-responsive vaccine delivery systems through the incorporation of $\mathrm{pH}$-responsive fusogenic polymers $[69,70]$. Synthetic fusogenic peptides with similar phase transition property have been wildly engaged in constructing $\mathrm{pH}$-responsive liposomes for various drug carriers, including those for cancer vaccines [71, 72]. Recently, Morishita et al. reported the surface-conjugation of exosomes derived from mouse B16F10 melanoma with GALA, a synthetic peptide containing 30 amino acids, to generate a $\mathrm{pH}$-responsive cancer vaccine named GALA-exo [54]. GALA-exo exhibited markedly enhanced MHC Class I antigen presentation compared with unmodified exosomes. Liposomes are typically superior to polymeric NPs in achieving higher loading capacity of hydrophilic antigens and faster release of vaccine cargos in the cytosol triggered by endogenous lipases. A potential limitation in applying $\mathrm{pH}$-responsive liposomes to cancer vaccine delivery in vivo is their relatively less stability in circulation or tissue interstitials, primarily due to 
easy disruption of the lipid membrane. Crosslinking of the lipid bilayer is a promising strategy to stabilize liposome vaccines without disturbing their property of antigen release [73].

Fusogenic peptides or polymers are also utilized for the modification of various natural or synthetic vaccine delivery systems for enhancing cytosol delivery of antigens. For example, Qiu et al. attached a $\mathrm{pH}$-sensitive peptide, named $\mathrm{pH}$ (low) insertion peptides (pHLIPs), onto the surface of a self-assembled NP vaccine via click chemistry to facilitate endosomal escape [74]. These pHLIP-containing NP vaccines (NP-pHLIP) loaded with a tumor-associated antigen NY-ESO-1 exhibited enhanced capability in inducing the activation and proliferation of antigen-specific $\mathrm{T}$ cells in vivo.

\subsubsection{3 pH-responsive biomaterials for "proton sponge" effect}

Polycations, e.g., polyamidoamine and polyethylenimine (PEI), possess substantial buffering capacity in the low $\mathrm{pH}$ environment of the endosome because of the high number of amine groups. This buffering capacity that leads to osmotic pressure change and endosome disruption, a phenomenon known as "proton sponge effect", has been extensively studied and explored for the application in cytosol delivery of nucleotide cargos [75]. Similarly, this effect could be utilized for promoting endosomal escape of cancer vaccines. In an elegant example, Gao and colleagues developed a library of ultra-pH-sensitive (UPS) NPs based on copolymers containing tertiary amines, which exhibited sharp response within narrow $\mathrm{pH}$ ranges (on/off within $\mathrm{pH}$ change of 0.25 ) [76]. Through in vivo screening of the capability in eliciting $\mathrm{CD} 8^{+}$ $\mathrm{T}$ cell response, PC7A NP, one of the UPS NPs, was selected as the promising candidate as a cancer vaccine carrier [35]. It is noticeable that PC7A NP potently activated DCs through activating stimulator of interferon genes (STING)-dependent pathways serving as both an antigen carrier and an adjuvant in this vaccine design. Such self-adjuvanted cancer vaccine delivered by PC7A mediated substantially improved inhibition of the growth of various tumors, including mouse melanoma, colorectal tumor, and human papilloma virus-E6/E7-infected tumor models in mice.

\subsubsection{Other $\mathrm{pH}$-responsive biomaterials}

Another interesting design of $\mathrm{pH}$-responsive cancer vaccine is to insert a $\mathrm{pH}$-responsive promoter that is co-delivered with other components of the vaccine. As an example, Liu et al. recently developed a novel endosome-disruptive delivery vehicle for vaccines by co-encapsulating a $\mathrm{pH}$-responsive promoter, i.e., ammonium bicarbonate $\left(\mathrm{NH}_{4} \mathrm{HCO}_{3}\right)$, and the vaccine cargo into a poly(D,L-lactic-co-glycolic acid) (PLGA) NP with a thin shell [34]. Upon internalization, the protons in the endosome reacted with $\mathrm{NH}_{4} \mathrm{HCO}_{3}$ to generate $\mathrm{NH}_{3}$ and $\mathrm{CO}_{2}$ gases, which facilitated endosomal escape of antigens by bursting the shell of NPs. This method using co-encapsulated $\mathrm{pH}$-responsive promoters provides a facile and versatile solution for controlling intracellular distribution of antigens. However, further studies are needed to elucidate the mechanism by which the released gases disrupt the endosomes and evaluate the potential cytotoxic effect of intracellular $\mathrm{NH}_{3}$ and $\mathrm{CO}_{2}$.

In summary, as illustrated by a number of examples above, extensive efforts have been undertaken to design and develop $\mathrm{pH}$-responsive biomaterials with diversified chemical structures for responsive cancer vaccine delivery utilizing the marked $\mathrm{pH}$ drop across the membrane of endosome. The key to success for this particular category of responsive biomaterials is the sensitivity to such $\mathrm{pH}$ change (from $\mathrm{pH} \sim 7$ to $\sim 5$ ). UPS NPs described above are a good example to demonstrate that ultra-sensitivity to $\mathrm{pH}$ change leads to substantially enhanced $\mathrm{CD} 8^{+} \mathrm{T}$ cell response and the resultant improved anticancer efficacy. Further efforts are necessary to improve the sensitivity of current $\mathrm{pH}$-responsive biomaterials to achieve more precise control of the antigen delivery for more effective cancer vaccines. In addition, facile and scalable chemical synthesis and preparation are always the key factors for potential clinical translation of biomaterials. Responsive biomaterials with scalability and welldefined preparation procedure are most likely to be tested in the clinical setting.

\subsubsection{Redox-responsive vaccine delivery system}

It has been long recognized that the eukaryotic cytoplasm exhibits significant reducibility compared 
to that of the extracellular environment, which is due to the extensive synthesis of glutathione (GSH), a tripeptide containing free thiol with reducing capability. This reductive small peptide is reproduced constantly by glutathione reductase to maintain low level of reactive oxygen species (ROS) in the cytoplasm, which is critical for normal metabolism in eukaryotes [77]. Similar to the low $\mathrm{pH}$ inside the endosome, the reducibility of cytoplasm has been widely utilized as an internal trigger to control the intracellular delivery of a variety of cargos, including antigens and adjuvants.

Designing redox-responsive vaccine delivery systems has mostly relied on the cleavage of a disulfide bond mediated by intracellular GSH. Typically, antigenic compounds are chemically conjugated onto the surface of a synthetic NP or the side-chains of a macromolecule through a disulfide-containing linkage. Hubbell and Swartz groups demonstrated the initial attempts to prepare a redox-responsive delivery system for cancer vaccines $[37,78]$. They conjugated OVA onto the surface of a polymeric NP with a reducible disulfide linkage. The redox-responsive vaccine elicited highly potent antigen-specific $\mathrm{CD} 8^{+} \mathrm{T}$ cell response compared with that by non-reducible vaccines or soluble antigens. The result is likely due to the redox-triggered release of antigen for endosomal escape and, thus, enhanced MHC Class I loading for cross-presentation.

The library of redox-responsive cancer vaccine delivery systems has been greatly enriched by engag- ing a variety of novel biomaterials with a reducible disulfide linkage, such as erythrocyte membraneenveloped polymeric NPs [26], $\beta$-glucan schizophyllan (SPG) complex [38], MOF [40], and nanogels [79]. In a recent example, Kramer et al. synthesized a reducible antigen-adjuvant conjugate with different sensitivities to precisely control the location of antigen release by employing the dramatic difference in reduction capability inside or outside DCs (Fig. 3) [39]. They designed two redox-responsive linkers with different redox sensitivities to prepare the conjugates by varying the chemical substituents adjacent to the disulfide bond (Fig. 3(a)). The more stable linker (HYN-SS) could be cleaved only intracellularly in a highly reducible environment, whereas the other linker (SS) could be readily cleaved even extracellularly with low reduction activity. Meanwhile, a non-reducible linker (HYN) was used as the control. The conjugates prepared with HYN-SS linker for intracellular antigen release outperformed the other two (SS and HYN) in protecting the immunized mice from tumor challenging (Figs. 3(b) and 3(c)). This study clearly revealed the importance of controlling the intracellular delivery and fate of antigens for enhanced antigen-specific $\mathrm{CD}^{+} \mathrm{T}$ cell response against cancer. In another elegant example, Wang et al. reported a "minimalist" nanovaccine $(\mathrm{mNV})$ by directly crosslinking the OVA proteins (with reduced free thiols) through disulfide linkage [41]. A CpG bearing a free thiol group was added to be

(a)

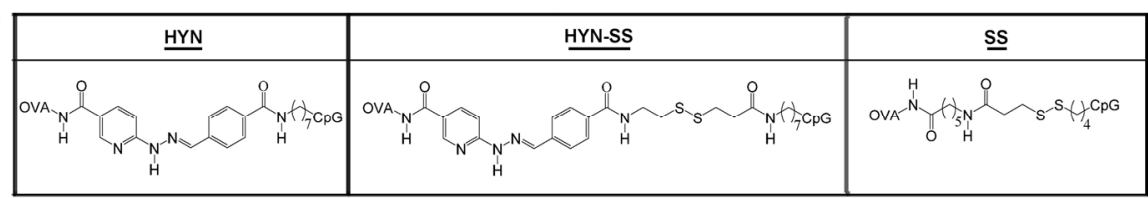

(b)

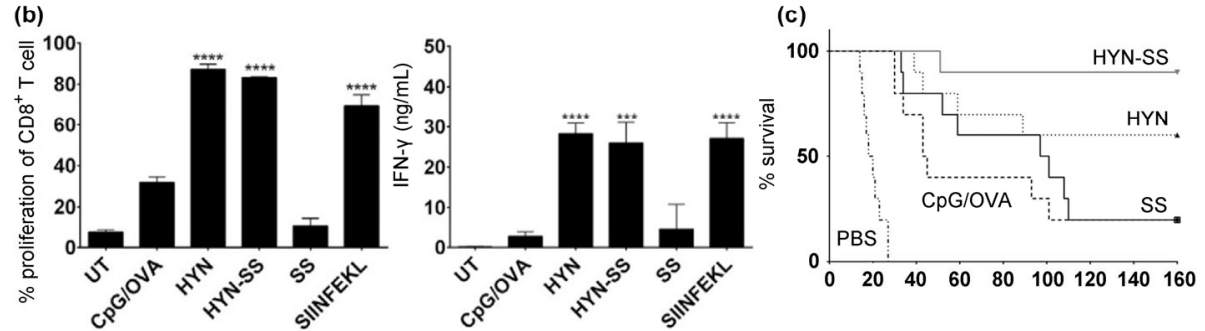

Figure 3 Comparison of different redox-cleavable linkers in cancer vaccines for eliciting cellular immune response against cancer. (a) Chemical structure of the chemical linkers for conjugating modified OVA antigen and $\mathrm{CpG}$ adjuvant. (b) $\mathrm{CD} 8^{+} \mathrm{T}$ cell proliferation and IFN- $\gamma$ secretion in the co-culture of bone marrow-derived dendritic cells pulsed with different conjugates in vitro. UT, untreated as the negative control; SIINFEKL, CD8 epitope peptide of OVA. (c) Survival rate of mice vaccinated with different conjugates and challenged with B16-OVA. Reproduced with permission from Ref. [39], C) Elseiver 2017. 
co-crosslinked with OVA. This type of vaccine, without involving any additional carrier materials, showed almost $100 \%$ loading capacity of antigen and adjuvant and elicited potent OVA-specific $\mathrm{CD}^{+} \mathrm{T}$ cell proliferation in vivo. Immunization with $\mathrm{mNV}$ significantly inhibited the growth of established B16-OVA melanoma in C57BL/6 mice and showed significant prophylactic efficacy on preventing the vaccinated mice against the tumor challenge. The novel "carrier-free" design of $\mathrm{mNV}$ maximized the loading capacity of antigen and adjuvant molecules and minimized the risk of toxicity and undesired immune response against carriers.

Compared with the diversified chemical design of $\mathrm{pH}$-responsive biomaterials, the design of redoxresponsive cancer vaccines is much more monotonous, primarily through the disulfide structure. It is noticeable that the acidic environment in endosomes/lysosomes may interfere with the cleavage reaction of the disulfide bond [80]. Thus, more redox-responsive biomaterials with innovative chemical design and increased sensitivity even in acidic environment are to be developed for cancer vaccine delivery. Combination of $\mathrm{pH}$ responsiveness for effective endosome escape and redox-responsiveness for accelerated release of antigens in cytosol could also be a promising direction.

\subsubsection{Light-responsive vaccine delivery system}

In addition to the internal triggers described above, some external triggers, such as light and radiation, have also been employed to construct stimuliresponsive vaccine delivery systems to achieve precise spatiotemporal control of antigen release. The general idea of designing light-responsive vaccine is to co-deliver a photosensitizer with the vaccine components within the carrier. When exposed to light of a certain wavelength, the photosensitizer is activated to initiate a photochemical reaction for generating considerable amount of ROS, which could disrupt the endosome membrane and subsequently facilitate the release and escape of antigens. This strategy is known as photochemical internalization (PCI). As an elegant example, Håkerud et al. engaged tetraphenyl chlorine disulfonate (TPCS2a), as a photosensitizer, to establish a series of light-responsive vaccine delivery systems, including soluble antigen complex $[42,81]$ and antigen-loaded liposomes [43]. The vaccinated mice that received irradiation with $435-\mathrm{nm}$ visible light exhibited remarkably enhanced $\mathrm{CD} 8^{+} \mathrm{T}$ cell response compared to that by the control groups without irradiation. This study suggested the critical role of PCI in controlling endosomal escape of antigen molecules and subsequent cross-priming of $\mathrm{CD} 8^{+} \mathrm{T}$ cells. In another example, Zhang et al. recently developed a well-defined light-responsive vaccine delivery system using Pheophorbide A (PheoA), a hydrophobic photosensitizer, grafted onto PEI [44]. In aqueous solution, PheoA-PEI self-assembled and formed a complex with the model antigen OVA through electrostatic interaction to generate a PheoA-PEI/OVA $\mathrm{NP}$ with the average diameter of $276 \mathrm{~nm}$ (Fig. 4(a)). Upon irradiation with light at the wavelength of $670 \mathrm{~nm}$, the NP could promote robust ROS generation for endosome disruption in DC2.4 cells. Subsequent release and intracellular delivery of the antigen triggered by light stimulation was confirmed by confocal laser scanning microscopy (CLSM) (Fig. 4(b)). Mice implanted with E.G7-OVA tumors were transdermally vaccinated around the tumor with DC2.4 cells, pulsed with PheoA-PEI/OVA NP, and irradiated with light of suitable wavelength before injection. Significant tumor inhibition was observed with the light-responsive NPs compared to that by the non-responsive NPs or free OVA. The role of photosensitizers in facilitating endosomal escape in cancer vaccine delivery is rather similar to that of $\mathrm{pH}$-responsive promoters. Further studies are necessary to exhibit more prominent advantage of using such photosensitizer-based light-responsive vaccine carriers in eliciting potent anticancer immune response.

Near-infrared (NIR) light penetrates the skin and tissue much deeper than lights of lower wavelengths due to the physiological transparent window [82]. Recently, Cao et al. developed a NIR-responsive vaccine carrier through loading antigen molecules and hyaluronic acid (HA) onto gold NPs (HA-OVAAuNPs) [45]. Surface bound HA facilitated CD44receptor-mediated cellular uptake of the nanovaccine with APCs. AuNP was employed to translate NIR radiation into thermal energy. After 3-min NIR laser irradiation, HA-OVA-AuNP-treated bone marrow DCs (BMDCs) exhibited substantially increased local 
(a)

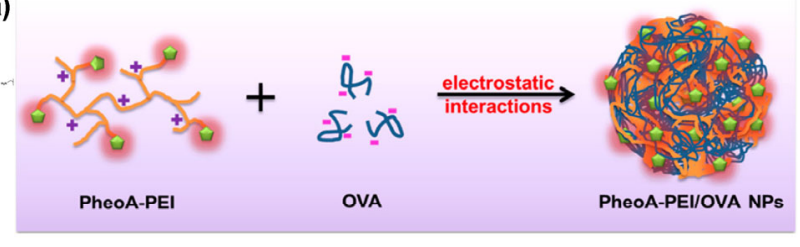

(b)

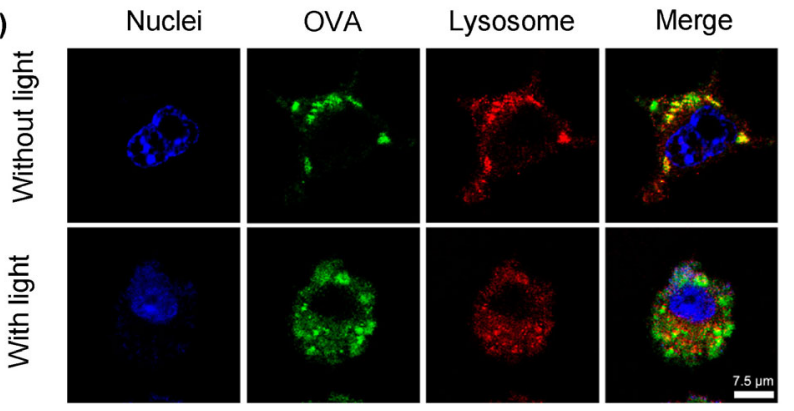

Figure 4 Light-responsive vaccine delivery system based on PheoA and PEI. (a) Schematic illustration of the formation of PheoA-PEI/OVA nanoparticles through self-assembly based on electrostatic interaction. (b) Confocal microscope images of DC2.4 cells pulsed with PheoA-PEI/OVA nanoparticles with or without light irradiation. Scale bar $=7.5 \mu \mathrm{m}$. Reproduced with permission from Ref. [44], (C) American Chemical Society 2017.

temperature that was as high as $42.3^{\circ} \mathrm{C}$. Such high local temperature facilitated the disruption of the endosome membrane for cytosol delivery of loaded antigen molecules and, thus, enhanced $\mathrm{CD}^{+} \mathrm{T}$ cell response. The improved $\mathrm{CD}^{+} \mathrm{T}$ cell proliferation led to markedly suppressed tumor growth in mice bearing EG.7-OVA tumor.

Light-responsive vaccine delivery systems utilizing well-controlled and defined external triggers provide advantages over systems relying on internal trigger, such as spatial control, by focusing on the activity of the released vaccine components at the selected area of tissues. An existing large library of available candidates of effective photosensitizers provides the necessary molecular basis for further development of this category of responsive biomaterials for cancer vaccine delivery [83]. Despite the achievements described above, light-responsive cancer vaccines are still in its early phase of development and more in vitro and in vivo studies are necessary to demonstrate its potential for clinical applications. In general, NIR-light responsive biomaterials have higher potential compared with UV- or visible light-responsive biomaterials for clinical applications due to deeper penetration in tissues.

\subsubsection{Other responsive vaccine delivery systems}

Some special responsiveness has been imparted to cancer vaccine delivery systems for enhanced uptake by APCs and cytosol delivery of vaccines beyond what has been described above. For example, some mechanical triggers can also be engaged to enhance antigen cross-presentation by accelerating the fusion of liposome vaccines with APC membrane. Un et al. demonstrated that ultrasound could be employed to enhance APC fusion with a DNA vaccine that was encapsulated in a PEGylated bubble lipoplex with mannose moieties conjugated on the surface $[46,47]$. This strategy, known as "sonoporation method", has been extensively employed for enhancing the efficiency of gene delivery. The mannose moieties facilitated anchoring of the liposomal vaccine on the surface of APCs through specific recognition by abundant mannose receptors of APCs. Subsequent ultrasound exposure led to the fusion of the bubble lipoplex with the cell membrane; the antigen-encoding plasmid DNA (pDNA) was, thus, directly delivered to the cytosol of APCs bypassing the endocytosis pathway. In a B16F10 melanoma mouse model, a pDNA vaccine elicited remarkably enhanced $C D 8^{+}$ $\mathrm{T}$ cell activation and cytokine secretion with the assistance of ultrasound, leading to significant tumor size inhibition and improved survival. Thus far, very few efforts have been made to control vaccine delivery using mechanical triggers. This elegant example using ultrasound provides a promising new direction for the future development of responsive cancer vaccines.

\section{Conclusion}

As illustrated by various examples, responsive biomaterials designed to respond to internal or external stimuli have exhibited great promise in enhancing cancer vaccines by eliciting robust and potent antigenspecific cellular immune responses. Although most efforts are focused on developing stimuli-responsive biomaterials to overcome the obstacles in intracellular delivery, very few examples of responsive biomaterials thus far are designed to modulate the biodistribution of cancer vaccines. Of all the responsive biomaterials applied in cancer vaccine delivery, $\mathrm{pH}$-responsive 
biomaterials are by far the most advanced, benefiting from several known endosome-disrupting mechanisms and the diverse design of the $\mathrm{pH}$-sensitive chemical moieties. As a comparison, redox- and photo-responsive materials are much less exploited but represent great promise in cancer vaccine delivery, given the highly reducing cytosolic environment and facile spatial control of light as an external trigger, respectively.

In the more general area of drug delivery using responsive biomaterials, other triggers that have been commonly employed, such as enzymes [84], electricity [85], and mechanical force [86], have yet to be explored in cancer vaccine delivery. For example, enzymes are an ideal trigger for stimuli-responsive delivery owing to their excellent efficiency and specificity, which has already been demonstrated extensively in the delivery of various drugs [84]. Applying enzyme-responsive biomaterials in cancer vaccine delivery is a promising new direction to pursue, considering the high levels of lipase in the cytoplasm, which may play a significant role in the degradation of some carriers, such as liposomes and polyester NPs [73].

There are multiple areas for the future of cancer vaccine development, wherein responsive biomaterials may play a significant role. Conventionally, most noncellular cancer vaccines are designed to target draining LNs through injections, including subcutaneous, intra-dermal, or intra-muscular. Although the spleen is the largest secondary lymphatic organ with high concentration of DCs, which are in close proximity with a high number of $\mathrm{T}$ cells, targeted delivery of cancer vaccine to spleen through intravenous (i.v.) injections is still challenging as these injection routes typically lead to relatively low immunogenicity and sever systemic side effects compared to that by other administration routes [87-89]. However, several recent studies have shown evidence that spleen could be an appealing target for self-adjuvanted cancer vaccines (vaccines without additional adjuvant molecules that are often toxic if administered systemically, e.g., mRNA vaccines, known for activating DCs themselves) [90, 91]. For example, a lipid complex with in vitro transcribed mRNA encoding neoantigens efficiently targeted

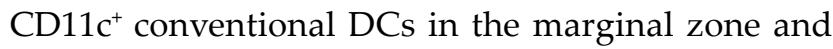
plasmacytoid DCs and macrophages in the spleen upon i.v. injection [91]. Remarkably high level of antigen-specific $\mathrm{CD}^{+} \mathrm{T}$ cell response (up to $30 \%-60 \%$ of the total $\mathrm{CD} 8^{+} \mathrm{T}$ cell population) has been achieved through fine-tuning the net charge of the RNA-lipid complex. Biomaterials, particularly responsive biomaterials, may play an important role in protecting mRNA/DNA vaccines from extracellular ribonucleases and promoting the uptake by APCs systemically.

Most cancer vaccines so for are designed to elicit $\mathrm{T}$ cell response. However, growing evidence has shown that B cells may also play an important role in potentiating antitumor immune response [92, 93]. B cell epitope-based cancer vaccines against a variety of tumor antigens, such as human epidermal growth factor receptor-2 (HER-2) [94], epidermal growth factor receptor (EGFR) [95], carcinoembryonic antigen (CEA) [96], and idiotype protein derived from B-cell malignancies [97], have also shown efficacy in preclinical and clinical studies. Responsive biomaterials are also expected to enhance the vaccination efficiency of this kind of vaccines for anticancer antibody response. Thus, the development of responsive biomaterials assisting B cell epitope-based cancer vaccines is a promising area to be explored in future.

Rapid advances have been made in the field of responsive biomaterials for cancer vaccines in the last two decades benefiting from both the increasing knowledge of cancer immunology and rapid progress in novel biomaterial design. It can be foreseen that more and more tailor-designed stimuli-responsive biomaterials will be developed to continually promote cancer vaccines. Although promising, there are several key challenges to be addressed to advance biomaterialassisted cancer vaccines for future clinical applications: (1) Biomaterials with sophisticated design have to meet with the requirements of scalability, facile and reproducible manufacturing, and high biocompatibility for potential clinical test. (2) Highly efficient loading of both the antigen and adjuvant represents another major challenge for improving the co-delivery of both vaccine components to the same APCs or even the same cellular compartment within an APC for optimized efficiency of antigen presentation [60]. (3) Sensitivity of the responsive biomaterials, in general, needs to be further improved to quantitatively control the location and dosage of the antigen and adjuvant. Novel chemistry and material design are 
expected to continually provide solutions for these challenges to facilitate the development of nextgeneration cancer vaccines.

\section{Acknowledgements}

This work was supported in part by the Foundation Pierre Mercier pour la science, ISREC Foundation with a donation from the Bateman Foundation, Swiss National Science Foundation (Project grant 315230_ 173243), Novartis Foundation for medical-biological Research (17A058), and the École polytechnique fédérale de Lausanne (EPFL).

\section{References}

[1] Melero, I.; Gaudernack, G.; Gerritsen, W.; Huber, C.; Parmiani, G.; Scholl, S.; Thatcher, N.; Wagstaff, J.; Zielinski, C.; Faulkner, I. et al. Therapeutic vaccines for cancer: An overview of clinical trials. Nat. Rev. Clin. Oncol. 2014, 11, 509-524.

[2] Cheever, M. A.; Higano, C. S. PROVENGE (sipuleucel-T) in prostate cancer: The first FDA-approved therapeutic cancer vaccine. Clin. Cancer Res. 2011, 17, 3520-3526.

[3] Sahin, U.; Türeci, Ö. Personalized vaccines for cancer immunotherapy. Science 2018, 359, 1355-1360.

[4] Zhu, G. Z.; Zhang, F. W.; Ni, Q. Q.; Niu, G.; Chen, X. Y. Efficient nanovaccine delivery in cancer immunotherapy. ACS Nano 2017, 11, 2387-2392.

[5] Lu, Z.-R.; Qiao, P. Drug delivery in cancer therapy, Quo Vadis? Mol. Pharmaceutics, in press, DOI: 10.1021/ acs.molpharmaceut.8b00037.

[6] Langer, R. Drug delivery and targeting. Nature 1998, 392, 5-10.

[7] Fenton, O. S.; Olafson, K. N.; Pillai, P. S.; Mitchell, M. J.; Langer, R. Advances in biomaterials for drug delivery. $A d v$. Mater. 2018, 30, 1705328.

[8] Luo, Z. C.; Wu, Q. J.; Yang, C. B.; Wang, H. M.; He, T.; Wang, Y. Z.; Wang, Z. Y.; Chen, H.; Li, X. Y.; Gong, C. Y. et al. A powerful $\mathrm{CD}^{+} \mathrm{T}$-cell stimulating D-tetra-peptide hydrogel as a very promising vaccine adjuvant. Adv. Mater. 2017, 29, 1601776.

[9] Irvine, D. J.; Hanson, M. C.; Rakhra, K.; Tokatlian, T. Synthetic nanoparticles for vaccines and immunotherapy. Chem. Rev. 2015, 115, 11109-11146.

[10] Mehta, N. K.; Moynihan, K. D.; Irvine, D. J. Engineering new approaches to cancer vaccines. Cancer Immunol. Res. 2015, 3, 836-843.
[11] Guo, Y. G.; Lei, K. W.; Tang, L. Neoantigen vaccine delivery for personalized anticancer immunotherapy. Front. Immunol. 2018, 9, 1499.

[12] Lu, Y.; Aimetti, A. A.; Langer, R.; Gu, Z. Bioresponsive materials. Nat. Rev. Mater. 2016, 2, 16075.

[13] Senapati, S.; Mahanta, A. K.; Kumar, S.; Maiti, P. Controlled drug delivery vehicles for cancer treatment and their performance. Signal Transduct. Target. Ther. 2018, 3, 7.

[14] Mura, S.; Nicolas, J.; Couvreur, P. Stimuli-responsive nanocarriers for drug delivery. Nat. Mater. 2013, 12, 991-1003.

[15] Nakayama, M.; Akimoto, J.; Okano, T. Polymeric micelles with stimuli-triggering systems for advanced cancer drug targeting. J. Drug Target. 2014, 22, 584-599.

[16] Pardoll, D. M. Cancer vaccines. Nature 1998, 4, 525-531.

[17] van der Burg, S. H.; Arens, R.; Ossendorp, F.; van Hall, T.; Melief, C. J. M. Vaccines for established cancer: Overcoming the challenges posed by immune evasion. Nat. Rev. Cancer 2016, 16, 219-233.

[18] Halle, S.; Halle, O.; Förster, R. Mechanisms and dynamics of $\mathrm{T}$ cell-mediated cytotoxicity in vivo. Trends Immunol. 2017, 38, 432-443.

[19] Garrido, F.; Aptsiauri, N.; Doorduijn, E. M.; Garcia Lora, A. M.; van Hall, T. The urgent need to recover MHC class I in cancers for effective immunotherapy. Curr. Opin. Immunol. 2016, 39, 44-51.

[20] Neefjes, J.; Jongsma, M. L. M.; Paul, P.; Bakke, O. Towards a systems understanding of MHC class I and MHC class II antigen presentation. Nat. Rev. Immunol. 2011, 11, 823-836.

[21] Kawai, T.; Akira, S. The role of pattern-recognition receptors in innate immunity: Update on toll-like receptors. Nat. Immunol. 2010, 11, 373-384.

[22] Sahdev, P.; Ochyl, L. J.; Moon, J. J. Biomaterials for nanoparticle vaccine delivery systems. Pharm. Res. 2014, $31,2563-2582$.

[23] Supersaxo, A.; Hein, W. R.; Steffen, H. Effect of molecular weight on the lymphatic absorption of water-soluble compounds following subcutaneous administration. Pharm. Res. 1990, 7, 167-169.

[24] Kaminskas, L. M.; Porter, C. J. H. Targeting the lymphatics using dendritic polymers (dendrimers). Adv. Drug Deliv. Rev. 2011, 63, 890-900.

[25] Reddy, S. T.; Rehor, A.; Schmoekel, H. G.; Hubbell, J. A.; Swartz, M. A. In vivo targeting of dendritic cells in lymph nodes with poly(propylene sulfide) nanoparticles. J. Control. Release 2006, 112, 26-34.

[26] Guo, Y. Y.; Wang, D.; Song, Q. L.; Wu, T. T.; Zhuang, X. T.; Bao, Y. L.; Kong, M.; Qi, Y.; Tan, S. W.; Zhang, Z. P. Erythrocyte membrane-enveloped polymeric nanoparticles 
as nanovaccine for induction of antitumor immunity against melanoma. ACS Nano 2015, 9, 6918-6933.

[27] Wang, C.; Ye, Y. Q.; Hu, Q. Y.; Bellotti, A.; Gu, Z. Tailoring biomaterials for cancer immunotherapy: Emerging trends and future outlook. Adv. Mater. 2017, 29, 1606036.

[28] Lynn, G. M.; Laga, R.; Darrah, P. A.; Ishizuka, A. S.; Balaci, A. J.; Dulcey, A. E.; Pechar, M.; Pola, R.; Gerner, M. Y.; Yamamoto, A. et al. In vivo characterization of the physicochemical properties of polymer-linked TLR agonists that enhance vaccine immunogenicity. Nat. Biotechnol. 2015, 33, 1201-1210.

[29] Kwon, Y. J.; James, E.; Shastri, N.; Fréchet, J. M. J. In vivo targeting of dendritic cells for activation of cellular immunity using vaccine carriers based on $\mathrm{pH}-$ responsive microparticles. Proc. Natl. Acad. Sci. USA 2005, 102, 18264-18268.

[30] Duan, F.; Feng, X. C.; Yang, X. J.; Sun, W. T.; Jin, Y.; Liu, H. F.; Ge, K.; Li, Z. H.; Zhang, J. C. A simple and powerful co-delivery system based on $\mathrm{pH}$-responsive metal-organic frameworks for enhanced cancer immunotherapy. Biomaterials 2017, 122, 23-33.

[31] Foster, S.; Duvall, C. L.; Crownover, E. F.; Hoffman, A. S.; Stayton, P. S. Intracellular delivery of a protein antigen with an endosomal-releasing polymer enhances CD8 T-cell production and prophylactic vaccine efficacy. Bioconjug. Chem. 2010, 21, 2205-2212.

[32] Wilson, J. T.; Keller, S.; Manganiello, M. J.; Cheng, C.; Lee, C. C.; Opara, C.; Convertine, A.; Stayton, P. S. pHresponsive nanoparticle vaccines for dual-delivery of antigens and immunostimulatory oligonucleotides. ACS Nano 2013, 7, 3912-3925.

[33] Yuba, E.; Kono, Y.; Harada, A.; Yokoyama, S.; Arai, M.; Kubo, K.; Kono, K. The application of $\mathrm{pH}$-sensitive polymer-lipids to antigen delivery for cancer immunotherapy. Biomaterials 2013, 34, 5711-5721.

[34] Liu, Q.; Chen, X. M.; Jia, J. L.; Zhang, W. F.; Yang, T. Y.; Wang, L. Y.; Ma, G. H. pH-responsive poly(D,L-lactic-coglycolic acid) nanoparticles with rapid antigen release behavior promote immune response. ACS Nano 2015, 9, 4925-4938.

[35] Luo, M.; Wang, H.; Wang, Z. H.; Cai, H. C.; Lu, Z. G.; Li, Y.; Du, M. J.; Huang, G.; Wang, C. S.; Chen, X. et al. A STING-activating nanovaccine for cancer immunotherapy. Nat. Nanotechnol. 2017, 12, 648-654.

[36] Morishita, M.; Takahashi, Y.; Nishikawa, M.; Ariizumi, R.; Takakura, Y. Enhanced class I tumor antigen presentation via cytosolic delivery of exosomal cargos by tumor-cell-derived exosomes displaying a $\mathrm{pH}$-sensitive fusogenic peptide. Mol. Pharmaceutics 2017, 14, 4079-4086.
[37] Eby, J. K.; Dane, K. Y.; O’Neil, C. P.; Hirosue, S.; Swartz, M. A.; Hubbell, J. A. Polymer micelles with pyridyl disulfide-coupled antigen travel through lymphatics and show enhanced cellular responses following immunization. Acta Biomater. 2012, 8, 3210-3217.

[38] Mochizuki, S.; Morishita, H.; Sakurai, K. Complex consisting of $\beta$-glucan and antigenic peptides with cleavage site for glutathione and aminopeptidases induces potent cytotoxic $\mathrm{T}$ lymphocytes. Bioconjugate Chem. 2017, 28, 2246-2253.

[39] Kramer, K.; Shields, N. J.; Poppe, V.; Young, S. L.; Walker, G. F. Intracellular cleavable $\mathrm{CpG}$ oligodeoxynucleotideantigen conjugate enhances anti-tumor immunity. Mol. Ther. 2017, 25, 62-70.

[40] Yang, Y.; Chen, Q. Q.; Wu, J.-P.; Kirk, T. B.; Xu, J. K.; Liu, Z. H.; Xue, W. Reduction-responsive codelivery system based on a metal-organic framework for eliciting potent cellular immune response. ACS Appl. Mater. Interfaces 2018, 10, 12463-12473.

[41] Wang, K.; Wen, S. M.; He, L. H.; Li, A.; Li, Y.; Dong, H. Q.; Li, W.; Ren, T. B.; Shi, D. L.; Li, Y. Y. "Minimalist" nanovaccine constituted from near whole antigen for cancer immunotherapy. ACS Nano 2018, 12, 6398-6409.

[42] Håkerud, M.; Waeckerle-Men, Y.; Selbo, P. K.; Kündig, T. M.; Høgset, A.; Johansen, P. Intradermal photosensitisation facilitates stimulation of MHC class-I restricted CD8 T-cell responses of co-administered antigen. J. Control. Release 2014, 174, 143-150.

[43] Hjálmsdóttir, Á.; Bühler, C.; Vonwil, V.; Roveri, M.; Håkerud, M.; Wäckerle-Men, Y.; Gander, B.; Johansen, P. Cytosolic delivery of liposomal vaccines by means of the concomitant photosensitization of phagosomes. Mol. Pharmaceutics 2016, 13, 320-329.

[44] Zhang, C. N.; Zhang, J.; Shi, G. N.; Song, H. J.; Shi, S. B.; Zhang, X. Y.; Huang, P. S.; Wang, Z. H.; Wang, W. W.; Wang, C. et al. A light responsive nanoparticle-based delivery system using pheophorbide A graft polyethylenimine for dendritic cell-based cancer immunotherapy. Mol. Pharmaceutics 2017, 14, 1760-1770.

[45] Cao, F. Q.; Yan, M. M.; Liu, Y. J.; Liu, L. X.; Ma, G. L. Photothermally controlled MHC class I restricted CD8 T-cell responses elicited by hyaluronic acid decorated gold nanoparticles as a vaccine for cancer immunotherapy. $A d v$. Healthc. Mater. 2018, 7, 1701439.

[46] Un, K.; Kawakami, S.; Suzuki, R.; Maruyama, K.; Yamashita, F.; Hashida, M. Suppression of melanoma growth and metastasis by DNA vaccination using an ultrasound-responsive and mannose-modified gene carrier. Mol. Pharmaceutics 2011, 8, 543-554. 
[47] Yoshida, M.; Kawakami, S.; Kono, Y.; Un, K.; Higuchi, Y.; Maruyama, K.; Yamashita, F.; Hashida, M. Enhancement of the anti-tumor effect of DNA vaccination using an ultrasoundresponsive mannose-modified gene carrier in combination with doxorubicin-encapsulated PEGylated liposomes. Int. J. Pharm. 2014, 475, 401-407.

[48] Reddy, S. T.; van der Vlies, A. J.; Simeoni, E.; Angeli, V.; Randolph, G. J.; O’Neil, C. P.; Lee, L. K.; Swartz, M. A.; Hubbell, J. A. Exploiting lymphatic transport and complement activation in nanoparticle vaccines. Nat. Biotechnol. 2007, 25, 1159-1164.

[49] Liu, H. P.; Moynihan, K. D.; Zheng, Y. R.; Szeto, G. L.; Li, A. V; Huang, B.; van Egeren, D. S.; Park, C.; Irvine, D. J. Structure-based programming of lymph-node targeting in molecular vaccines. Nature 2014, 507, 519-522.

[50] Jewell, C. M.; Bustamante López, S. C.; Irvine, D. J. In situ engineering of the lymph node microenvironment via intranodal injection of adjuvant-releasing polymer particles. Proc. Natl. Acad. Sci. USA 2011, 108, 15745-15750.

[51] Trombetta, E. S.; Ebersold, M.; Garrett, W.; Pypaert, M.; Mellman, I. Activation of lysosomal function during dendritic cell maturation. Science. 2003, 299, 1400-1403.

[52] Overly, C. C.; Lee, K. D.; Berthiaume, E.; Hollenbeck, P. J. Quantitative measurement of intraorganelle $\mathrm{pH}$ in the endosomal-lysosomal pathway in neurons by using ratiometric imaging with pyranine. Proc. Natl. Acad. Sci. USA 1995, 92, 3156-3160.

[53] Murthy, N.; Thng, Y. X.; Schuck, S.; Xu, M. C.; Fréchet, J. M. J. A novel strategy for encapsulation and release of proteins: Hydrogels and microgels with acid-labile acetal cross-linkers. J. Am. Chem. Soc. 2002, 124, 12398-12399.

[54] Murthy, N.; Xu, M. C.; Schuck, S.; Kunisawa, J.; Shastri, N.; Fréchet, J. M. J. A macromolecular delivery vehicle for protein-based vaccines: Acid-degradable protein-loaded microgels. Proc. Natl. Acad. Sci. USA 2003, 100, 4995-5000.

[55] Cohen, J. A.; Beaudette, T. T.; Tseng, W. W.; Bachelder, E. M.; Mende, I.; Engleman, E. G.; Fréchet, J. M. J. T-cell activation by antigen-loaded $\mathrm{pH}$-sensitive hydrogel particles in vivo: The effect of particle size. Bioconjug. Chem. 2009, 20, 111-119.

[56] Bachelder, E. M.; Beaudette, T. T.; Broaders, K. E.; Paramonov, S. E.; Dashe, J.; Fréchet, J. M. J. Acid-degradable polyurethane particles for protein-based vaccines: Biological evaluation and in vitro analysis of particle degradation products. Mol. Pharmaceutics 2008, 5, 876-884.

[57] Ruff, L. E.; Mahmoud, E. A.; Sankaranarayanan, J.; Morachis, J. M.; Katayama, C. D.; Corr, M.; Hedrick, S. M.; Almutairi, A. Antigen-loaded pH-sensitive hydrogel micro- particles are taken up by dendritic cells with no requirement for targeting antibodies. Integr. Biol. 2013, 5, 195-203.

[58] Nuhn, L.; Vanparijs, N.; De Beuckelaer, A.; Lybaert, L.; Verstraete, G.; Deswarte, K.; Lienenklaus, S.; Shukla, N. M.; Salyer, A. C. D.; Lambrecht, B. N. et al. pH-degradable imidazoquinoline-ligated nanogels for lymph node-focused immune activation. Proc. Natl. Acad. Sci. USA 2016, 113, 8098-8103.

[59] van Herck, S.; van Hoecke, L.; Louage, B.; Lybaert, L.; De Coen, R.; Kasmi, S.; Esser-Kahn, A. P.; David, S. A.; Nuhn, L.; Schepens, B. et al. Transiently thermoresponsive acetal polymers for safe and effective administration of amphotericin B as a vaccine adjuvant. Bioconjug. Chem. 2018, 29, 748-760.

[60] Magarian Blander, J.; Medzhitov, R. Toll-dependent selection of microbial antigens for presentation by dendritic cells. Nature 2006, 440, 808-812.

[61] Maier, K.; Wagner, E. Acid-labile traceless click linker for protein transduction. J. Am. Chem. Soc. 2012, 134, 1016910173.

[62] Gupta, P.; Vermani, K.; Garg, S. Hydrogels: From controlled release to $\mathrm{pH}$-responsive drug delivery. Drug Discov. Today 2002, 7, 569-579.

[63] van der Maaden, K.; Varypataki, E. M.; Romeijn, S.; Ossendorp, F.; Jiskoot, W.; Bouwstra, J. Ovalbumin-coated $\mathrm{pH}$-sensitive microneedle arrays effectively induce ovalbuminspecific antibody and T-cell responses in mice. Eur. J. Pharm. Biopharm. 2014, 88, 310-315.

[64] Tirrell, D. A.; Takigawa, D. Y.; Seki, K. pH sensitization of phospholipid vesicles via complexation with synthetic poly(carboxylic acid). Ann. N. Y. Acad. Sci. 1985, 446, 237-248.

[65] Flanary, S.; Hoffman, A. S.; Stayton, P. S. Antigen delivery with poly(propylacrylic acid) conjugation enhances MHC-1 presentation and T-cell activation. Bioconjugate Chem. 2009, 20, 241-248.

[66] Keller, S.; Wilson, J. T.; Patilea, G. I.; Kern, H. B.; Convertine, A. J.; Stayton, P. S. Neutral polymer micelle carriers with $\mathrm{pH}$-responsive, endosome-releasing activity modulate antigen trafficking to enhance $\mathrm{CD}^{+} \mathrm{T}$ cell responses. J. Control. Release 2014, 191, 24-33.

[67] Yuba, E.; Harada, A.; Sakanishi, Y.; Kono, K. Carboxylated hyperbranched poly(glycidol)s for preparation of $\mathrm{pH}$-sensitive liposomes. J. Control. Release 2011, 149, 72-80.

[68] Arab, A.; Behravan, J.; Razazan, A.; Gholizadeh, Z.; Nikpoor, A. R.; Barati, N.; Mosaffa, F.; Badiee, A.; Jaafari, M. R. A nano-liposome vaccine carrying E75, a HER-2/neu-derived peptide, exhibits significant antitumour activity in mice. $J$. Drug Target. 2018, 26, 365-372. 
[69] Yuba, E. Design of pH-sensitive polymer-modified liposomes for antigen delivery and their application in cancer immunotherapy. Polym. J. 2016, 48, 761-771.

[70] Yuba, E.; Sakaguchi, N.; Kanda, Y.; Miyazaki, M.; Koiwai, K. pH-responsive micelle-based cytoplasmic delivery system for induction of cellular immunity. Vaccines 2017, 5, 41.

[71] Hatakeyama, H.; Ito, E.; Akita, H.; Oishi, M.; Nagasaki, Y.; Futaki, S.; Harashima, H. A pH-sensitive fusogenic peptide facilitates endosomal escape and greatly enhances the gene silencing of siRNA-containing nanoparticles in vitro and in vivo. J. Control. Release 2009, 139, 127-132.

[72] Li, W. J.; Nicol, F.; Szoka, F. C. GALA: A designed synthetic pH-responsive amphipathic peptide with applications in drug and gene delivery. Adv. Drug Deliv. Rev. 2004, 56, 967-985.

[73] Moon, J. J.; Suh, H.; Bershteyn, A.; Stephan, M. T.; Liu, H. P.; Huang, B.; Sohail, M.; Luo, S.; Ho Um, S.; Khant, H. et al. Interbilayer-crosslinked multilamellar vesicles as synthetic vaccines for potent humoral and cellular immune responses. Nat. Mater. 2011, 10, 243-251.

[74] Qiu, L. P.; Valente, M.; Dolen, Y.; Jäger, E.; ter Beest, M.; Zheng, L. Y.; Figdor, C. G.; Verdoes, M. Endolysosomalescape nanovaccines through adjuvant-induced tumor antigen assembly for enhanced effector $\mathrm{CD}^{+} \mathrm{T}$ cell activation. Small 2018, 14, 1703539.

[75] Boussif, O.; Lezoualc'h, F.; Zanta, M. A.; Mergny, M. D.; Scherman, D.; Demeneix, B.; Behr, J. P. A versatile vector for gene and oligonucleotide transfer into cells in culture and in vivo: Polyethylenimine. Proc. Natl. Acad. Sci. USA 1995, 92, 7297-7301.

[76] Ma, X. P.; Wang, Y. G.; Zhao, T.; Li, Y.; Su, L. C.; Wang, Z. H.; Huang, G.; Sumer, B. D.; Gao, J. M. Ultra-pH-sensitive nanoprobe library with broad $\mathrm{pH}$ tunability and fluorescence emissions. J. Am. Chem. Soc. 2014, 136, 11085-11092.

[77] López-Mirabal, H. R.; Winther, J. R. Redox characteristics of the eukaryotic cytosol. Biochim. Biophys. Acta - Mol. Cell Res. 2008, 1783, 629-640.

[78] Bearinger, J. P.; Terrettaz, S.; Michel, R.; Tirelli, N.; Vogel, H.; Textor, M.; Hubbell, J. A. Chemisorbed poly(propylene sulphide)-based copolymers resist biomolecular interactions. Nat. Mater. 2003, 2, 259-264.

[79] Li, D. D.; Sun, F. L.; Bourajjaj, M.; Chen, Y. N.; Pieters, E. H.; Chen, J.; van den Dikkenberg, J. B.; Lou, B.; Camps, M. G. M.; Ossendorp, F. et al. Strong in vivo antitumor responses induced by an antigen immobilized in nanogels via reducible bonds. Nanoscale 2016, 8, 19592-19604.

[80] Go, Y. M.; Jones, D. P. Redox compartmentalization in eukaryotic cells. Biochim. Biophys. Acta - Gen. Subj. 2008, 1780, 1273-1290.
[81] Håkerud, M.; Selbo, P. K.; Waeckerle-Men, Y.; Contassot, E.; Dziunycz, P.; Kündig, T. M.; Høgset, A.; Johansen, P. Photosensitisation facilitates cross-priming of adjuvant-free protein vaccines and stimulation of tumour-suppressing CD8 T cells. J. Control. Release 2015, 198, 10-17.

[82] Weissleder, R.; Ntziachristos, V. Shedding light onto live molecular targets. Nat. Med. 2003, 9, 123-128.

[83] Ji, Y.; Zhao, J. H.; Chu, C. C. Enhanced MHC-I antigen presentation from the delivery of ovalbumin by lightfacilitated biodegradable poly(ester amide)s nanoparticles. J. Mater. Chem. B 2018, 6, 1930-1942.

[84] Hu, Q. Y.; Katti, P. S.; Gu, Z. Enzyme-responsive nanomaterials for controlled drug delivery. Nanoscale 2014, 6 , 12273-12286.

[85] Balint, R.; Cassidy, N. J.; Cartmell, S. H. Conductive polymers: Towards a smart biomaterial for tissue engineering. Acta Biomater. 2014, 10, 2341-2353.

[86] Zhang, Y. Q.; Yu, J. C.; Bomba, H. N.; Zhu, Y.; Gu, Z. Mechanical force-triggered drug delivery. Chem. Rev. 2016, 116, 12536-12563.

[87] Ramsay, J. D.; Williams, C. L.; Simko, E. Fatal adverse pulmonary reaction in calves after inadvertent intravenous vaccination. Vet. Pathol. 2005, 42, 492-495.

[88] Reichmuth, A. M.; Oberli, M. A.; Jaklenec, A.; Langer, R.; Blankschtein, D. mRNA vaccine delivery using lipid nanoparticles. Ther. Deliv 2016, 7, 319-334.

[89] Zhang, L.; Wang, W.; Wang, S. X. Effect of vaccine administration modality on immunogenicity and efficacy. Expert Rev. Vaccines 2015, 14, 1509-1523.

[90] Broos, K.; van der Jeught, K.; Puttemans, J.; Goyvaerts, C.; Heirman, C.; Dewitte, H.; Verbeke, R.; Lentacker, I.; Thielemans, K.; Breckpot, K. Particle-mediated intravenous delivery of antigen mRNA results in strong antigen-specific T-cell responses despite the induction of type I interferon. Mol. Ther. Nucleic Acids 2016, 5, e326.

[91] Kranz, L. M.; Diken, M.; Haas, H.; Kreiter, S.; Loquai, C.; Reuter, K. C.; Meng, M.; Fritz, D.; Vascotto, F.; Hefesha, H. et al. Systemic RNA delivery to dendritic cells exploits antiviral defence for cancer immunotherapy. Nature 2016, 534, 396-401.

[92] Nelson, B. H. CD $20^{+}$B cells: The other tumor-infiltrating lymphocytes. J. Immunol. 2010, 185, 4977-4982.

[93] Germain, C.; Gnjatic, S.; Tamzalit, F.; Knockaert, S.; Remark, R.; Goc, J.; Lepelley, A.; Becht, E.; Katsahian, S.; Bizouard, G. et al. Presence of B cells in tertiary lymphoid structures is associated with a protective immunity in patients with lung cancer. Am. J. Respir. Crit. Care Med. 2014, 189, 832-844. 
[94] Riemer, A. B.; Untersmayr, E.; Knittelfelder, R.; Duschl, A.; Pehamberger, H.; Zielinski, C. C.; Scheiner, O.; JensenJarolim, E. Active induction of tumor-specific IgE antibodies by oral mimotope vaccination. Cancer Res. 2007, 67, 3406-3412.

[95] Hartmann, C.; Müller, N.; Blaukat, A.; Koch, J.; Benhar, I.; Wels, W. S. Peptide mimotopes recognized by antibodies cetuximab and matuzumab induce a functionally equivalent anti-EGFR immune response. Oncogene 2010, 29, 4517-4527.

[96] Brämswig, K. H.; Knittelfelder, R.; Gruber, S.; Untersmayr,
E.; Riemer, A. B.; Szalai, K.; Horvat, R.; Kammerer, R.; Zimmermann, W.; Zielinski, C. C. et al. Immunization with mimotopes prevents growth of carcinoembryonic antigen positive tumors in BALB/c mice. Clin Cancer Res. 2007, 13, 6501-6509.

[97] Ng, P. P.; Jia, M.; Patel, K. G.; Brody, J. D.; Swartz, J. R.; Levy, S.; Levy, R. A vaccine directed to B cells and produced by cell-free protein synthesis generates potent antilymphoma immunity. Proc. Natl. Acad. Sci. USA 2012, 109, 14526-14531. 